\title{
Macroscopic flow disequilibrium over aeolian dune fields
}

A. Gunn ${ }^{1}$, P. Schmutz ${ }^{2}$, M. Wanker ${ }^{3}$, D. A. Edmonds ${ }^{3}$, R. C. Ewing ${ }^{4}$, D. J. Jerolmack $^{1,5}$

\footnotetext{
${ }^{1}$ Department of Earth \& Environmental Science, University of Pennsylvania, Philadelphia, PA 19104, USA

${ }^{2}$ Department of Earth \& Environmental Science, University of West Florida, Pensacola, FL 32514, USA ${ }^{3}$ Department of Earth \& Atmospheric Sciences, Indiana University, Bloomington, IN 47405, USA

${ }^{4}$ Department of Geology \& Geophysics, Texas A\&M University, College Station, TX 77843, USA

${ }^{5}$ Department of Mechanical Engineering \& Applied Mechanics, University of Pennsylvania, Philadelphia, PA 19104, USA
}

\section{Key Points:}

- Wind and sediment flux decrease downwind from the leading edge of White Sands Dune Field

- A Lagrangian 1D model explains how non-equilibrium flow induced by surface roughness transitions produces observed dynamics

- Results are generalized using the 1D model for other roughness scenarios 


\begin{abstract}
Aeolian dune fields are self-organized patterns formed by wind-blown sand. Dunes are topographic roughness elements that impose drag on the atmospheric boundary layer (ABL), creating a natural coupling between form and flow. While the steady-state influence of drag on the ABL is well studied, non-equilibrium effects due to roughness transitions are less understood. Here we examine the large-scale coupling between the ABL and an entire dune field. Field observations at White Sands, New Mexico, reveal a concomitant decline in wind speed and sand flux downwind of the transition from smooth playa to rough dunes at the upwind dune-field margin, that affects the entire $\sim 10-\mathrm{km}$ long dune field. Using a simple theory for the system that accounts for the observations, we generalize to other roughness scenarios. We find that, via transitional ABL dynamics, aeolian sediment aggradation can be influenced by roughness both inside and outside dune fields.
\end{abstract}

\title{
Plain Language Summary
}

Just as how cyclists opt to have smooth skin to gain a speed advantage, near-surface winds in the atmosphere are faster over smooth topography. Dunes slow winds down because they are rough, but these features are also shaped by the winds. When wind passes over a boundary between smooth and rough surfaces, it can't instantaneously slow down; instead there is a transition zone where it decelerates. This area of transition can exist at the edge of a dune field if the land upwind of it is relatively smooth. We observe this at White Sands Dune Field, and generalize the results with theory. Aside from helping to explain the striking patterns we see on planetary surfaces, this result is useful because dunes are often used to interpret wind conditions.

\section{Introduction}

Aeolian dune fields are patterned areas of sand that express an aerodynamic coupling between the Earth's erodible surface and the atmospheric boundary layer (ABL) (Bagnold, 1941; Kocurek \& Ewing, 2005). For a single dune, this coupling is clear: flow accelerates on the stoss which erodes sand, and sand deposits on the lee where flow separates (Pye \& Tsoar, 2008). For a pair of dunes, the flow wake generated by an upwind dune influences the flow over a dune downwind, creating short-range interaction (Bristow et al., 2019; Bacik et al., 2020). For many dunes, the sum of their wakes imparts a collective drag (Stevens et al., 2015), creating long-range interaction. When there is a separation of scales between the bodies imparting drag and the flow, we parameterize this long-range interaction with the roughness length $z_{0}$ (Nikuradse, 1950; Stull, 2012; Nield et al., 2013). This is done extensively for dune-sized bodies in the ABL when flow is in equilibrium with the surface below (Garratt, 1990). Dune fields have edges, however, and the mismatch of roughness imparted by the dunes and the external surface can lead to a breakdown of equilibrium conditions downwind of the edge. This transient phenomena is rarely studied in geomorphology (Bauer, 1991; Jerolmack et al., 2012).

In the field of boundary layer meteorology, however, there has been significant work on how ABL flow changes due to a Lagrangian transition in surface properties; be it heat, water vapor flux, or roughness (Garratt, 1990; Bou-Zeid et al., 2004; Dupont \& Brunet, 2009). Observations, empirical relations and numerical models all describe a common pattern: when flow meets an edge between two surfaces, there is some transient disequilibrium between flow and the surface downwind of the edge. While this work has many valuable applications, in urban weather and renewable energy for example (Barlow, 2014; Stevens et al., 2015), none of these efforts have focused on flow crossing a dune-field boundary. This is a notable case, however, since the flow creates the form, which in turn alters the flow. 

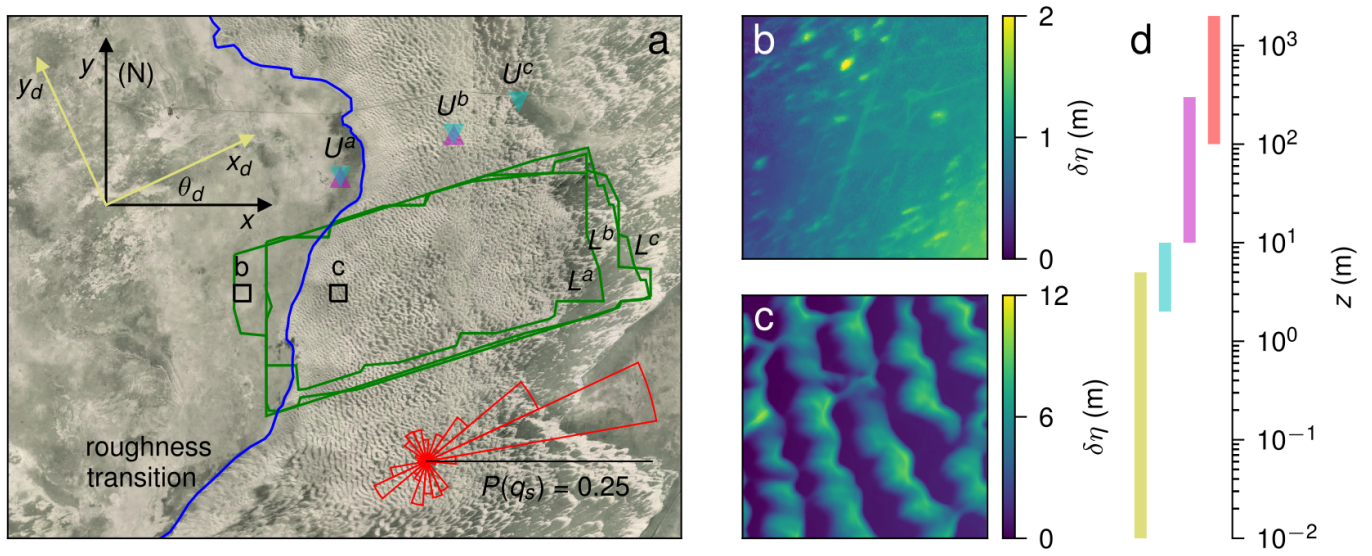

Figure 1. (a) A composite aerial photograph of White Sands Dune Field, New Mexico, annotated with; three topographic lidar dataset boundaries in green $\left(L^{a}, L^{b}, L^{c}\right)$, three ABL measurement locations $\left(U^{a}, U^{b}, U^{c}\right)$ for meteorological towers (cyan arrows) and doppler lidar (magenta arrows), the roughness transition delineating the 'smooth' playa and dune field (blue line), two pairs of axes $[x, y]$ (black, cardinal) and $\left[x_{d}, y_{d}\right]$ (yellow) that are offset by the dune direction $\theta_{d}$. Axes lines are $5 \mathrm{~km}$ centered on $\left[32.854^{\circ},-106.369^{\circ}\right]$. A sediment flux probability rose calculated from the tower at $U^{a}$ (red), black line gives scale. (b,c) $500 \mathrm{~m}^{2}$ sub-DTMs (locations in black in (a)) contrast playa and dune topography (note the different color-scales of elevation relative to minimum, $\delta \eta$ ). (d) The range of elevations for dune-field topography (yellow), meteorological tower observations (cyan), wind lidar (magenta), and ABL height (red).

Jerolmack et al. (2012) presented the idea of an internal boundary layer developing over the White Sands Dune Field in New Mexico, USA. This dune field has a unidirectional sediment flux (Figs. 1a \& S1) and a well-defined dune-field boundary, where a consistently 'smooth' upwind playa transitions to active 'rough' dunes (McKee, 1966; Kocurek et al., 2007). Jerolmack et al. (2012) hypothesized that the transition creates flow deceleration across the dune field, causing measured changes in dune migration that control vegetation density and even the groundwater table. They did not make direct wind observations, however; in lieu of this, Jerolmack et al. (2012) drew upon empirical closures from classical boundary-layer studies that seldom apply to the ABL. Furthermore, since their study, alternative hypotheses for the observed dune migration patterns at White Sands have arisen that dispute the influence of the roughness transition (Baitis et al., 2014; Pelletier, 2015). To resolve this conflict, we apply a recent transient ABL theory (Momen \& Bou-Zeid, 2016) to White Sands, finding the wind adjustment due to roughness change and the associated lengthscale. We then present measurements of winds and topography at White Sands (Fig. 1) that agree with the theoretical prediction. We examine near-surface winds at three locations on a stream-wise transect, winds up to $300 \mathrm{~m}$ at two locations (Gunn et al., 2018), and dune migration patterns from three topographic surveys. Our work shows that at White Sands the ABL is out-of-equilibrium with the dune roughness creating stream-wise gradients in sediment flux. Finally, with theory we suggest this mechanism is significant for most dune fields on Earth. 


\section{Materials and Methods}

\subsection{ABL theory}

We first seek to develop a physically-informed expectation of the flow adjustment at the leading edge of a dune field. Models of the ABL cover a broad range of complexity. Often, computationally expensive Large Eddy Simulations (LES) are chosen to understand the temporal response of the ABL to forcing. The richness of each LES experiment, however, comes at the expense of limiting the number of forcings examined. Instead we chose to employ an analytical model extending from Momen and Bou-Zeid (2016), opting for the ability to generalize and interpret. Such a model seems appropriate also given the limited data available for comparison.

The main assumption of this model is that the turbulent stresses responsible for mixing momentum vertically in the ABL are linearly proportional to the local horizontal flow speed. Using a Reynolds decomposition where $u=\bar{u}+u^{\prime}$,

$$
\frac{\partial\left(u^{\prime} w^{\prime}\right)}{\partial z} \simeq \alpha \bar{u} .
$$

Here $u$ is horizontal wind speed, $w$ is vertical wind speed, $z$ is altitude, and $\alpha$ is a damping frequency that is proportional to how easily momentum is transferred. An identical equation is assumed for the orthogonal horizontal wind speed $v$. We further assume that the ABL is neutral, barotropic and locally planar-homogeneous. Then, if we let $A=$ $u+i v$, where $i$ is the imaginary unit, and set the geostrophic wind vector to $A_{g}=G+$ $i \cdot 0$ the ABL flow equation is:

$$
\dot{A}=i f(G-A)-\alpha A,
$$

where the over-dot indicates change in time and $f$ is the Coriolis frequency. Steady-state flow therefore takes the form $A=G /(1-i \alpha / f)$.

Now consider the case where a Lagrangian column of air that is in equilibrium, $A_{\text {out }}$, encounters a step-change in environment characterized by $\alpha_{i n}$ at $t=0$ such that it tends toward a new equilibrium $A_{i n}$; the solution to Eqn. 2 is:

$$
A=\underbrace{x A_{\text {out }}}_{\text {homogeneous }}+\underbrace{(1-x) A_{\text {in }}}_{\text {particular }}
$$

where $x=e^{-t\left(\alpha_{i n}+i f\right)}$. Assuming Taylor's hypothesis, we can switch from a Lagrangian reference frame to an Eulerian one. A parcel in the column travels with the flow $U$ where $U=|A|$, such that $t=S / U$ where $S$ is the stream-wise distance from the step-change in environment.

Using Eq. 3 in an Eulerian reference frame, we can describe the transience that is caused by flow encountering a change in roughness between the inside and outside of a dune field - all that is required is a model that links momentum with roughness via $\alpha$. Here we choose the following model used by others (Momen \& Bou-Zeid, 2016; Lentz, 2001; Herrmann \& Madsen, 2007):

$$
\alpha=\frac{A_{g} L_{0}}{\log \left(z / z_{0}\right) Z^{2}}
$$

where there are three characteristic length-scales: $L_{0}$ for turbulent mixing, $z_{0}$ for roughness, and $Z$ for ABL height. To apply Eqs. 3 and 4 , we choose values for the free parameters $L_{0}, Z, z_{0, \text { out }}, z_{0, \text { in }}$, and $f$. Following verification from LES (Momen \& BouZeid, 2016) we choose $L_{0}=50 \mathrm{~m}$. At White Sands the average ABL height is approximately $Z=1000 \mathrm{~m}$ (Hinds \& Hoidale, 1977) (red line Fig. 1d), and the latitude constrains $f=7.91 \cdot 10^{-5} \mathrm{~s}^{-1}$. We assume $z_{0, \text { out }}=10^{-4} \mathrm{~m}$ for the playa and $z_{0, \text { in }}=$ 
$10^{-1} \mathrm{~m}$ for the dune field. These follow typical values for boundary layer studies of similar landscapes (Stull, 2012), and the classical result from hydraulically rough flow where $z_{0}=k_{s} / 30$ and $k_{s}$ is the element height (a ripple and a dune for upwind and downwind, respectively, Fig 1d) (Nikuradse, 1950). With these elements in place, we are able to predict the magnitude and distance of the expected downwind decrease in wind speed at White Sands.

\subsection{ABL observations}

We observed the winds at White Sands Dune Field using two separate systems: a set of three 10-m meteorological towers measuring near-surface concurrently, and a single upward-facing doppler wind lidar measuring wind profiles at heights $10 \mathrm{~m} \leq z \leq$ $300 \mathrm{~m}$ at two separate locations on consecutive years. The periods of observation for all instruments overlapped with the 'windy season', in which strong southwesterly winds emanating from the Pacific cross New Mexico between February and June.

The three meteorological towers were erected in 2015 along a transect approximately in the 'formative' wind direction (cyan triangles at $U^{a}, U^{b}$, and $U^{c}$ in Fig. 1a, respectively). These towers measured wind speeds at 2, 5 and $10 \mathrm{~m}$ elevation, and wind direction at $10 \mathrm{~m}$ elevation, using cup and vein anemometers (cyan line Fig. 1d). Data were stored for the 10-minute means. The harsh environment at White Sands Dune Field (heat waves, dust storms, lightning, etc.) has limited when these towers were able to measure all variables concurrently, to 160 days. Only these periods are reported here.

A Zephir300 lidar recorded data adjacent to Tower A for 59 days in 2016, and to Tower B for 18 days in 2017, and was used to measure the winds between 10 and 300 m elevation (magenta triangles at $U^{a}$ and $U^{b}$ in Fig. 1a, respectively). Means for 17-s intervals at ten elevations between 10 and $300 \mathrm{~m}$ (magenta line Fig. 1d) were taken. Here we only report the horizontal wind speeds.

\subsection{Topography observations}

We analyze three White Sands Dune Field terrestrial airborne lidar survey DTMs to understand the dune direction and sediment fluxes. These surveys are publicly available on OpenTopography.org and were taken in January 2009, September 2009, and June $2010\left(L^{a}, L^{b}\right.$, and $L^{c}$ in Fig. 1a, respectively). Dune direction, $\theta_{d}$, was found by analyzing the slip-face orientations (Pedersen et al., 2015; Swanson et al., 2016) using a crosscorrelation technique (Supporting Text S1, Fig. S1). Sediment flux, $q_{s}$, was found by integrating the Exner equation,

$$
q_{s}(s)=q_{s}\left(s_{0}\right)-\phi \int_{s_{0}}^{s} \frac{d \eta}{d t} d x_{d}
$$

Where $\phi$ is the packing fraction, $s$ is some distance along a line of constant $y_{d}, d \eta$ is a change in elevation over some time interval $d t$, and $q_{s}(s)$ is the sediment flux $q_{s}$ at some location $s$. If $q_{s}\left(s_{0}\right)$ is known, then $q_{s}(s)$ can be found by performing the integral on the RHS of Eq. 5 (Supporting Text S1, Figs. S2 \& S3).

\section{Results}

The impact of the roughness transition can be seen in both form and flow at White Sands (Fig. 2). To illustrate the difference in topographic variability that induces flow drag, we plot the span-wise average transect in standard deviation over a $100 \mathrm{~m}^{2}$ movingwindow (yellow line). The playa outside the dune field, $S_{d}<0$, is smooth relative to the dunes, which can also be seen in an example elevation transect (grey background in Fig. 2). Average downwind flow deceleration in 10-m wind is shown for concurrent Met tower measurements (cyan in Fig. 2). These speeds are filtered such that for a given time, 


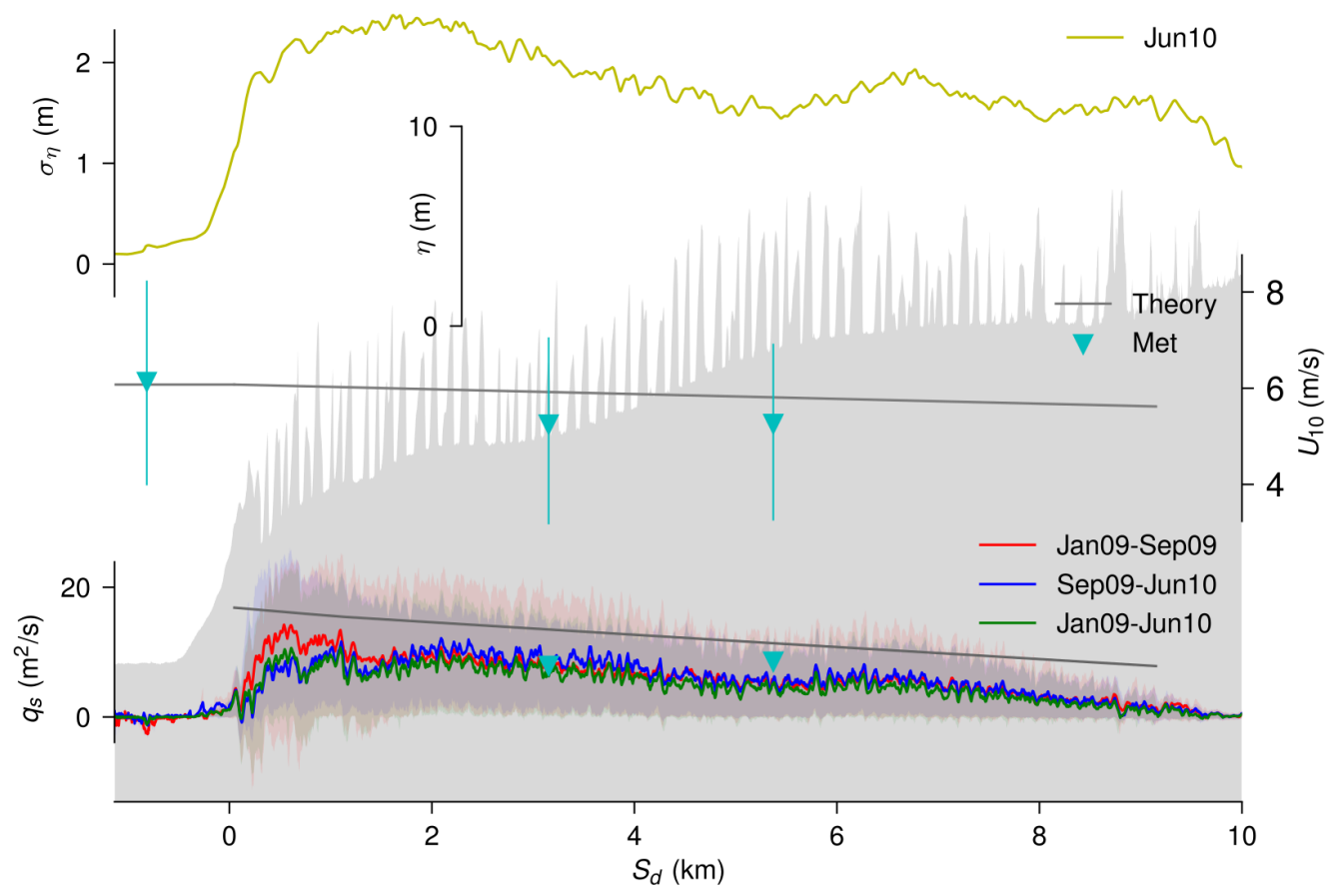

Figure 2. White Sands transects in $S_{d}$, the stream-wise direction where the roughness transition is at the origin. Bumpy shaded background is an example DTM elevation, $\eta$, transect illustrating the roughness transition and net deposition. The span-wise mean local topographic standard deviation (yellow line), $\sigma_{\eta}$, shown here for the June 2010 DTM, is low upwind of the margin and consistently high downwind. This causes a deceleration in $10-\mathrm{m}$ wind, $U_{10}$, shown for theory (upper grey line) and concurrent observations (cyan; triangles, average; lines, interquartile range). In turn, the sediment flux, $q_{s}$, diminishes downwind, as measured explicitly from DTM pairs (red, blue and green; lines, span-wise average; envelopes, span-wise interquartile range) and calculated from theory (lower grey line) and dune-aligned 10-m winds (cyan triangles). Sediment flux calculated with $U_{10}^{a}$ is not shown due to limited sediment supply on the playa. 

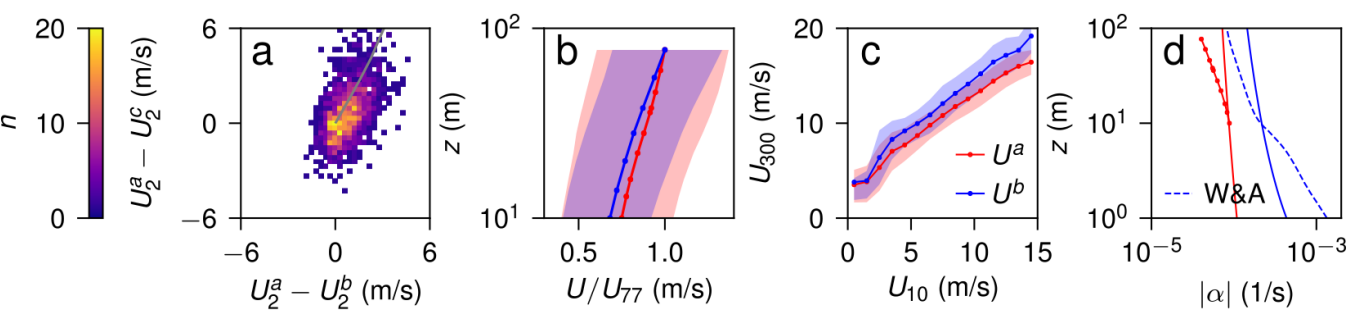

Figure 3. (a) A histogram of residuals between concurrent 2-m wind speeds, $U_{2}^{a}, U_{2}^{b}$ and $U_{2}^{c}$, at the three meteorological towers, with theory (grey line) overlaid. (b) Mean doppler lidar wind speed profiles between 10 and $77 \mathrm{~m}$ (for $\theta_{10} \in\left\{\theta_{d} \pm 15^{\circ}\right\}$ ) from the playa $\left(U^{a}\right.$, red line) and within the dune field ( $U^{b}$, blue line), normalized and plotted with interquartile range envelopes. (c) Mean (lines) and interquartile ranges (envelopes) of $U_{300}$ given $U_{10}$. (d) Theoretical profiles of the damping frequency, $\alpha$, for the playa (red) and the dune field (blue) for a fixed geostrophic condition, and inferred profiles from the playa doppler lidar (red dotted line) and LES simulations by Wang and Anderson (2019) (blue dashed line).

wind direction is within $\theta_{d} \pm n \cdot 15^{\circ}$ where $n=\{1,2,3\}$ for $U_{a}, U_{b}$ and $U_{c}$, respectively, meaning flow is approximately aligned with the dunes everywhere. The idealized model (upper grey line in Fig. 2) gives fair agreement with the observed deceleration. This theory line is the weighted average of an ensemble of lines with different $G$ values such that it has the same observed probability of $U_{10}^{a}$ values. This averaging is important because flow deceleration is non-linearly related to the upwind speed (Eq. 2). The coupling between flow and form is then closed by sediment flux, which we illustrate in three independent calculations (bottom Fig. 2). Explicit measurement of sediment flux comes from DTM pairs, which show transport diminishing downwind. We compare this to sediment flux calculations determined from measured $U_{10}$ winds at met towers $U^{b}$ and $U^{c}$ (for $\theta \in$ $\left.\left\{\theta_{d} \pm 15^{\circ}\right\}\right)$, using the following relations linking $U_{10}$ and $q_{s}$ :

$$
\begin{aligned}
U_{10} & =\frac{u_{*}}{\kappa} \log \left(\frac{10}{z_{0}}\right) \\
q_{s} & =\frac{9 \rho_{\text {air }}}{5 g \rho_{\text {sand }}} u_{*, c r} u_{*}^{2}\left(1-{\frac{u_{*, c r}}{u_{*}}}^{2}\right),
\end{aligned}
$$

where $u_{*}$ is the friction velocity, $\kappa$ is von Karman's constant, $\rho$ is the density of the subscripts, $g$ is gravity, and $u_{*, c r}$ is the critical friction velocity above which sediment transport is initiated. Eq. 6 has been used elsewhere (Kok et al., 2012; Durán et al., 2011). Our choice of $u_{*, c r}=0.3 \mathrm{~m} / \mathrm{s}$ is within the range previously used at White Sands (Reitz et al., 2010; Jerolmack et al., 2011; Eastwood et al., 2012); $z_{0}$ used in this flux calculation is $10^{-4} \mathrm{~m}$, since wind at the saltation scale 'feels' the ripple-scale drag, consistent with other studies from White Sands (Jerolmack et al., 2006; Martin et al., 2013; Gunn et al., 2018). The chosen parameter values predict a flux decline that reasonably follows the DTM-derived trend, though offset by a constant factor (bottom Fig. 2). Other parameter values or flux relations could perhaps be chosen to better 'fit' the data, but none would influence the overall trend of downwind-decreasing sand flux.

The roughness transition at White Sands also affects ABL flow structure (Fig. 3). To show the downwind evolution of near-surface wind, residuals of aligned concurrent wind speeds measured by the towers are plotted in Figure 3a. Consistent with theory, observations prefer the NNE octant of this space such that $U_{2}^{a}>U_{2}^{b}>U_{2}^{c}$. The magnitudes of these residuals are large enough to indicate that sediment flux sometimes occurs at upwind locations, but not downwind, in the dune field. Doppler lidar flow pro- 

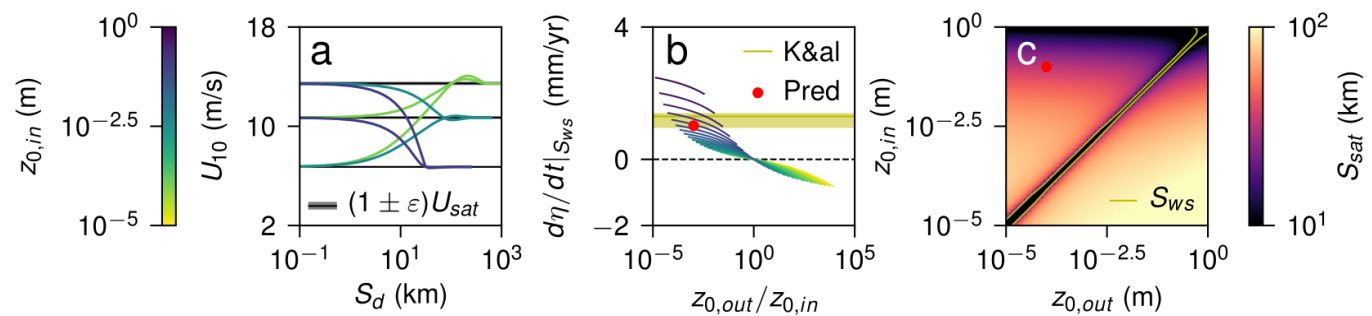

Figure 4. (a) Theoretical scenarios of the evolution of $10-\mathrm{m}$ wind speed $\left(U_{10}\right)$ with distance downwind $\left(S_{d}\right)$ of a transition from roughness lengths of $z_{0, \text { out }}$ to $z_{0, \text { in }}$. Line color indicates $z_{0, \text { in }}$ from the left color-bar, and black lines indicate equilibrium speeds, $U_{\text {sat }}$ (small grey envelopes are within $1 \%$ of $U_{\text {sat }}$. (b) Theoretical deposition rates, $d \eta / d t$, at distance $S_{w s}$ downwind of the transition for a given ratio of outside and inside (line color) $z_{0}$. Overlaid is the prediction for White Sands (red dot) and measured deposition rate (yellow; line, average; envelope, interquartile range) using optical thermo-luminescence dating by Kocurek et al. (2007). (c) The; theoretical distance $S_{\text {sat }}$ downwind of a roughness transition to achieve $U_{10}$ that is in equilibrium with $z_{0, i n}$, where color has a log-scale. Stream-wise length of White Sands Dune Field at the measured wind transect is $S_{w s}=5 \mathrm{~km}$ (yellow line); and the assumed White Sands $z_{0, \text { out }}$ and $z_{0, \text { in }}$ (red dot), are shown.

files far above the dunes are also altered substantially for dune-aligned winds, such that for a given speed aloft the near-surface shear is diminished inside the dune field relative to outside (Fig. 3b). In turn, this ensures that flow aloft must be greater over the dune field to achieve the same near-surface speed, with this compensation growing with magnitude (Fig. 3c). The damping frequency $\alpha$ paramaterizes how much shear-induced turbulent mixing there is given mean flow speed (Eq. 1). Theoretical profiles of $\alpha$ consistent with Momen and Bou-Zeid $(2016,2017)$ for the constants at White Sands and $G=$ $20 \mathrm{~m} / \mathrm{s}$ (Jerolmack et al., 2012) are compared to profiles inferred from assumed-equilibrium flows in Figure $3 \mathrm{~d}$. The inferred $\alpha$ profiles are found using wind profiles and rearranging Eq. 3 with $\dot{A}=0$. This assumption, that flow is locally steady, should hold for the average profile measured by the doppler wind lidar at $U^{a}$, and an LES simulation over a periodic sub-domain of White Sands dunes performed by Wang and Anderson (2019). The lidar and LES should be compared to the theoretical profiles outside and inside the dune field, respectively. They show that the parameterization for $\alpha$ in Eq. 4 underestimates the vertical gradient in damping but is consistent at $10 \mathrm{~m}$, where the ABL theory is tied to sediment flux with Eq. 6.

The results from White Sands indicate that the roughness disparity at the dunefield boundary has geomorphic consequences. This could occur in dune fields with different dune and external roughness lengths. We broaden our results by varying roughness lengths inside and outside a hypothetical region in the idealized theory with $G=$ $20 \mathrm{~m} / \mathrm{s}$ (Fig. 4). This exercise approximates the isolated coupling of flow and the erodible form that impinges upon it. In Figure 4a, example cases of non-equilibrium flow speeds downwind of a transition from $z_{0, \text { out }}$ to $z_{0, \text { in }}$ are shown. The trajectories highlight that equilibrium depends uniquely on the local roughness, but the approach depends on both roughness values (Eq. 3). By Eq. 5, convergence of sediment causes deposition, meaning that in a uni-directional wind regime, a decrease (increase) in flux with distance downwind causes aggradation (degradation) in the dune field. Plotting the deposition rate a fixed distance from the roughness transition (here the stream-wise length of White Sands dune field, $S_{w s}$ ) for different pairs of roughness lengths illustrates that for domains rougher inside than outside, deposition occurs inside because flow is decelerating (Fig. 4b). For the pair of roughness lengths assumed for White Sands, the predicted aggradation rate 
is within the interquartile range measured by Kocurek et al. (2007) of 0.9-1.4 mm/yr, when the flux intermittency factor $(0.05)$ is chosen so the upwind flux matches Fig. 2 . The distance downwind $S_{\text {sat }}$ needed to achieve equilibrium between $z_{0, \text { in }}$ and $U_{10}$ is defined graphically as the distance $S_{d}$ such that $U_{10}$ is first within $1 \%$ of $U_{\text {sat }}$ (grey bands in Fig. 4a). This length-scale is shown for roughness length pairs in Figure 4c, with $S_{\text {sat }}$ for White Sands (32 km, red dot) far exceeding $S_{w s}$ (5 km, yellow line), implying that flow across the entire White Sands dune field is out of equilibrium.

\section{Discussion}

Our interpretation of the White Sands results is that the majority of the downwind decrease in sediment flux, and associated dune migration rate, can be related to the roughness transition at the dune field boundary. While these dynamics were anticipated by Jerolmack et al. (2012), our work provides: (i) the first direct wind measurements demonstrating flow adjustment; (ii) application of a more physically-informed model; and (iii) an improved treatment of sand flux. We suspect that the flow disequilibrium extends well beyond the dune field at White Sands, as indicated by the theoretical results $\left(S_{w s} \ll\right.$ $S_{\text {sat }}$ ) and the observed aggradation across the entire dune field (Fig. 2). We also expect that the observed downwind gradients in vegetation density, grain-size, dune morphology and water-table depth at White Sands (Jerolmack et al., 2012; Ewing \& Kocurek, 2010; Lee et al., 2019) would all be dramatically different if the upwind conditions were altered.

Limitations exist on the application and interpretation of this work. The influence of roughness transitions on flow can be outweighed by other land-ABL interactions, notably the exchange of heat can dominate ABL flow profiles on short timescales (Stull, 2012). Indeed, at White Sands flow profiles are extremely sensitive to the diurnal cycle (Gunn et al., 2018; Frank \& Kocurek, 1994); however, spatially we expect the impact of these properties to be small relative to that of the roughness. Flow direction and the dune-field boundary are clear at White Sands, but other cases are less conducive to this form of analysis. Finally, flow that is out of equilibrium with the surface outside a dune field will not behave as outlined here as it evolves over the dunes. This case probably exists for some dune fields, however we are confident that Eq. 3 applies at White Sands, and the observations of flow deceleration and profile alteration are robust.

More generally, this macroscopic length-scale of flow and form disequilibrium, $S_{\text {sat }}$, can be considered alongside other fundamental lengths in aeolian landscape evolution and is similar to $L_{\text {sat }}$, the saturation length of saltation that sets the dune instability wavelength (Durán et al., 2011). $S_{\text {sat }}$ can be considered the minimum length a dune field must be to attain equilibrium with the ABL. An important distinction, however, is that $S_{\text {sat }}$ depends on conditions outside the dune field as well as those within (highlighted by the diagonal asymmetry in Fig. 4c). Dune interaction and migration within $S_{\text {sat }}$ of the dune-field windward edge is fundamentally different from when the ABL is equilibrated with the macroscopic dune roughness. Based on the relative length of $S_{\text {sat }}$ values (Fig 4c) and typical dune-field lengths $\left(10^{1}-10^{3} \mathrm{~km}\right)$, we hypothesize that a significant portion of Earth's dunes exist within this out-of-equilibrium edge of dune fields (also suggested by Gao et al. (2015)).

Interestingly, the theory suggests that the flux and wind vector directions also change alongside their length inside $S_{\text {sat }}$ due to the evolving importance of drag relative to geostrophy (Fig. S4). White Sands does not have sufficient stream-wise extent to observe this effect (a $22^{\circ}$ deflection at equilibrium $S_{\text {sat }}=32 \mathrm{~km}$, in theory); however, in larger domains the dune direction may be affected (suggested by Warren (1976)). All else equal, deflection would increase with latitude, akin to other large Rossby-number dependent geomorphic forms like turbidity current channels (Wells \& Cossu, 2013). Another untested indication from this theory lies in the evolution of incipient dune fields where the cou- 
pling between $z_{0, i n}$ and $q_{s}$ is most sensitive. As dunes develop, a dune field may switch from net erosion to deposition if its roughness crosses the external roughness (right to left in Fig. 4b). Future tests of this idea could extend to the relative effects of heat flux and roughness transitions at coastal dune fields, or where the surface upwind of a unidirectional dune field has coarse span-wise heterogeneity.

\section{Acknowledgments}

Code to reproduce this paper and wind data are here https://github.com/algunn/ dune-impinge. Topography data is hosted by OpenTopography here http://opentopo .sdsc. edu/datasets?search=white $\backslash \% 20$ sands. We are very grateful for David Bustos' continuing facilitation of research at White Sands National Monument. We thank Scott R. David and Kieran B.J. Dunne for field assistance, Elie Bou-Zeid for useful discussions, and Gary Kocurek for topography data (D.O.I.s 10.5069/G9ZK5DMD and 10.5069/G9Q23X5P). Funding provided by; National Science Foundation (NSF) NRI INT award \#1734355 to D.J.J.; White Sands National Monument through NPS-GC-CESU Cooperative Agreement \#P12AC51051 and NSF through the National Center for Airborne Laser Mapping's EAR award \#1043051 for topography data (D.O.I. 10.5069/G97D2S2D) to R.C.E; International Society of Aeolian Research through the Elsevier Aeolian Research Scholarship to A.G; and UPenn Earth and Environmental Science department in support of the GEOL305 class.

CRediT: Conceptualization, Resources, Writing - review \& editing, A.G., D.A.E., R.C.E., D.J.J.; Data curation, Formal analysis, Software, Validation, Visualization, Writing - original draft; A.G.; Supervision, D.J.J.; Project administration, D.A.E., R.C.E., D.J.J.; Investigation, Methodology, all authors.

\section{References}

Bacik, K. A., Lovett, S., Colm-cille, P. C., \& Vriend, N. M. (2020). Wake induced long range repulsion of aqueous dunes. Physical Review Letters, 124(5), 054501.

Bagnold, R. A. (1941). The physics of blown sand and desert dunes. Methuen.

Baitis, E., Kocurek, G., Smith, V., Mohrig, D., Ewing, R. C., \& Peyret, A.-P. (2014). Definition and origin of the dune-field pattern at white sands, new mexico. Aeolian Research, 15, 269-287.

Barlow, J. F. (2014). Progress in observing and modelling the urban boundary layer. Urban Climate, 10, 216-240.

Bauer, B. O. (1991). Aeolian decoupling of beach sediments. Annals of the Association of American Geographers, 81 (2), 290-303.

Bou-Zeid, E., Meneveau, C., \& Parlange, M. B. (2004). Large-eddy simulation of neutral atmospheric boundary layer flow over heterogeneous surfaces: blending height and effective surface roughness. Water Resources Research, 40(2).

Bristow, N., Blois, G., Best, J. L., \& Christensen, K. (2019). Spatial scales of turbulent flow structures associated with interacting barchan dunes. Journal of Geophysical Research: Earth Surface, 124(5), 1175-1200.

Dupont, S., \& Brunet, Y. (2009). Coherent structures in canopy edge flow: a largeeddy simulation study. Journal of Fluid Mechanics, 630, 93-128.

Durán, O., Claudin, P., \& Andreotti, B. (2011). On aeolian transport: Grain-scale interactions, dynamical mechanisms and scaling laws. Aeolian Research, 3(3), 243-270.

Eastwood, E. N., Kocurek, G., Mohrig, D., \& Swanson, T. (2012). Methodology for reconstructing wind direction, wind speed and duration of wind events from aeolian cross-strata. Journal of Geophysical Research: Earth Surface, $117(\mathrm{~F} 3)$.

Ewing, R. C., \& Kocurek, G. A. (2010). Aeolian dune interactions and dune-field 
pattern formation: White sands dune field, new mexico. Sedimentology, 57(5), 1199-1219.

Frank, A., \& Kocurek, G. (1994). $\quad$ Effects of atmospheric conditions on wind profiles and aeolian sand transport with an example from white sands national monument. Earth Surface Processes and Landforms, 19(8), 735-745.

Gao, X., Narteau, C., \& Rozier, O. (2015). Development and steady states of transverse dunes: A numerical analysis of dune pattern coarsening and giant dunes. Journal of Geophysical Research: Earth Surface, 120(10), 2200-2219.

Garratt, J. (1990). The internal boundary layer-a review. Boundary-Layer Meteorology, 50(1-4), 171-203.

Gunn, A., Wanker, M., Lancaster, N., Edmonds, D. A., Ewing, R. C., \& Jerolmack, D. J. (2018). Circadian rhythm of dune-field activity. arXiv preprint arXiv:1812.03612.

Herrmann, M. J., \& Madsen, O. S. (2007). Effect of stratification due to suspended sand on velocity and concentration distribution in unidirectional flows. Journal of Geophysical Research: Oceans, 112(C2).

Hinds, B., \& Hoidale, G. (1977). Boundary layer dust occurrence iv atmospheric dust over selected geographical areas (Tech. Rep.). White Sands Missile Range, New Mexico: Army Electronics Command, Atmospheric Sciences Lab.

Jerolmack, D. J., Ewing, R. C., Falcini, F., Martin, R. L., Masteller, C., Phillips, C., ... Buynevich, I. (2012). Internal boundary layer model for the evolution of desert dune fields. Nature Geoscience, 5(3), 206-209.

Jerolmack, D. J., Mohrig, D., Grotzinger, J. P., Fike, D. A., \& Watters, W. A. (2006). Spatial grain size sorting in eolian ripples and estimation of wind conditions on planetary surfaces: Application to meridiani planum, mars. Journal of Geophysical Research: Planets, 111 (E12).

Jerolmack, D. J., Reitz, M. D., \& Martin, R. L. (2011). Sorting out abrasion in a gypsum dune field. Journal of Geophysical Research: Earth Surface, 116(F2).

Kocurek, G., Carr, M., Ewing, R., Havholm, K. G., Nagar, Y., \& Singhvi, A. (2007). White sands dune field, new mexico: age, dune dynamics and recent accumulations. Sedimentary Geology, 197(3-4), 313-331.

Kocurek, G., \& Ewing, R. C. (2005). Aeolian dune field self-organizationimplications for the formation of simple versus complex dune-field patterns. Geomorphology, 72(1-4), 94-105.

Kok, J. F., Parteli, E. J., Michaels, T. I., \& Karam, D. B. (2012). The physics of wind-blown sand and dust. Reports on progress in Physics, 75 (10), 106901.

Lee, D. B., Ferdowsi, B., \& Jerolmack, D. J. (2019). The imprint of vegetation on desert dune dynamics. Geophysical Research Letters, 46(21), 12041-12048.

Lentz, S. J. (2001). The influence of stratification on the wind-driven cross-shelf circulation over the north carolina shelf. Journal of Physical Oceanography, $31(9), 2749-2760$.

Martin, R. L., Barchyn, T. E., Hugenholtz, C. H., \& Jerolmack, D. J. Timescale dependence of aeolian sand flux observations under atmospheric turbulence. Journal of Geophysical Research: Atmospheres, 118(16), 9078 9092.

McKee, E. D. (1966). Structures of dunes at white sands national monument, new mexico (and a comparison with structures of dunes from other selected areas). Sedimentology, 7(1), 3-69.

Momen, M., \& Bou-Zeid, E. (2016). Large-eddy simulations and damped-oscillator models of the unsteady ekman boundary layer. Journal of the Atmospheric Sciences, 73(1), 25-40.

Momen, M., \& Bou-Zeid, E. (2017). Analytical reduced models for the nonstationary diabatic atmospheric boundary layer. Boundary-Layer Meteorology, $164(3), 383-399$.

Nield, J. M., King, J., Wiggs, G. F., Leyland, J., Bryant, R. G., Chiverrell, R. C., 
.. others (2013). Estimating aerodynamic roughness over complex surface terrain. Journal of Geophysical Research: Atmospheres, 118(23), 12-948.

Nikuradse, J. (1950). Laws of flow in rough pipes (Tech. Rep.). Washington, D.C.: National Advisory Committee for Aeronautics.

Pedersen, A., Kocurek, G., Mohrig, D., \& Smith, V. (2015). Dune deformation in a multi-directional wind regime: White sands dune field, new mexico. Earth Surface Processes and Landforms, 40(7), 925-941.

Pelletier, J. D. (2015). Controls on the large-scale spatial variations of dune field properties in the barchanoid portion of white sands dune field, new mexico. Journal of Geophysical Research: Earth Surface, 120(3), 453-473.

Pye, K., \& Tsoar, H. (2008). Aeolian sand and sand dunes. Springer Science \& Business Media.

Reitz, M. D., Jerolmack, D. J., Ewing, R. C., \& Martin, R. L. (2010). Barchanparabolic dune pattern transition from vegetation stability threshold. Geophysical Research Letters, $37(19)$.

Stevens, R. J., Gayme, D. F., \& Meneveau, C. (2015). Coupled wake boundary layer model of wind-farms. Journal of renewable and sustainable energy, 7(2), 023115 .

Stull, R. B. (2012). An introduction to boundary layer meteorology (Vol. 13). Springer Science \& Business Media.

Swanson, T., Mohrig, D., \& Kocurek, G. (2016). Aeolian dune sediment flux variability over an annual cycle of wind. Sedimentology, 63(6), 1753-1764.

Wang, C., \& Anderson, W. (2019). Turbulence coherence within canonical and realistic aeolian dune-field roughness sublayers. Boundary-Layer Meteorology, $173(3), 409-434$.

Warren, A. (1976). Dune trend and the ekman spiral. Nature, 259(5545), 653-654.

Wells, M., \& Cossu, R. (2013). The possible role of coriolis forces in structuring large-scale sinuous patterns of submarine channel-levee systems. Philosophical Transactions of the Royal Society A: Mathematical, Physical and Engineering Sciences, 371 (2004), 20120366. 


\section{Supporting Information for "Macroscopic flow disequilibrium over aeolian dune-fields"}

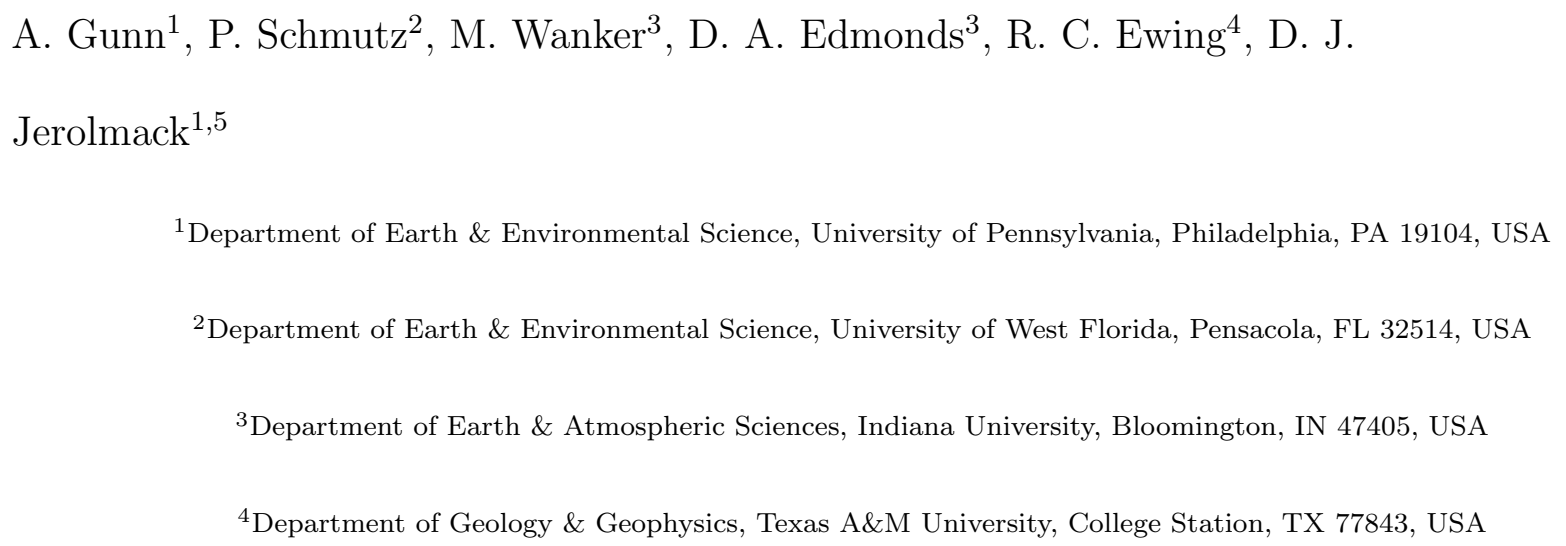

${ }^{1}$ Department of Earth \& Environmental Science, University of Pennsylvania, Philadelphia, PA 19104, USA

${ }^{2}$ Department of Earth \& Environmental Science, University of West Florida, Pensacola, FL 32514, USA

${ }^{3}$ Department of Earth \& Atmospheric Sciences, Indiana University, Bloomington, IN 47405, USA

${ }^{4}$ Department of Geology \& Geophysics, Texas A\&M University, College Station, TX 77843, USA

${ }^{5}$ Department of Mechanical Engineering \& Applied Mechanics, University of Pennsylvania, Philadelphia, PA 19104, USA

\section{Contents of this file}

1. Text S1

2. Figures $\mathrm{S} 1$ to $\mathrm{S} 4$

Introduction This file includes supporting figures (Figs. S1-S4) for the main paper. It also includes a section (Text S1) providing a more detailed methodology for the topographic data analysis. 
Text S1. Digital Terrain Models (DTMs) with 1-m horizontal resolution were produced from the three point clouds by taking the minimum elevation point within each DTM pixel as its elevation $\eta$. A first set of DTMs was produced on the cardinal bases to find the dune direction. A second set of DTMs were then produced on the span-wise and stream-wise bases to find the sediment flux (Fig. S2). Each set of three DTMs used for either orientation or flux used a common pixel grid.

Slip faces are the steepest slopes on the landscape and close to the angle of repose. They were isolated in the DTMs as pixels where the slope exceeded $30^{\circ}$. The full DTM was segmented into sub-tiles of $500 \mathrm{~m}^{2}$ to find the distribution of $\theta_{d}$ (Fig. S1). The peak of a cross-correlation between the distribution of the slip-face orientations, $P\left(\theta_{s f}\right)$, and $P\left(-\theta_{s f}\right)$ was taken as the dune migration direction for each sub-tile (Fig. S2). This method was used as an alternative to the mean or mode slip-face orientation of a sub-tile, for example, since those can be biased by the radial and bilateral asymmetry in dunes (i.e. slipfaces are mostly on dune arms, which can also be different lengths).

Using a common grid DTM, with $x_{d}$ positive in the dune direction and $y_{d}$ orthogonal, lines of adjacent pixels with constant $y_{d}$ in a pair of DTMs can be used to approximate sediment flux. This assumes that sediment flux is only in the direction of dunes, which is reasonable at White Sands Dune Field (Figs. 1a \& S1). The Exner equation can be written as,

$$
q_{s}(s)=q_{s}\left(s_{0}\right)-\phi \int_{s_{0}}^{s} \frac{d \eta}{d t} d x_{d}
$$

Where $\phi$ is the packing fraction, $s$ is some distance along a line of constant $y_{r}, d \eta$ is a change in elevation over some time interval $d t$, and $q_{s}(s)$ is the sediment flux $q_{s}$ at some 
location $s$. If $q_{s}\left(s_{0}\right)$ is known, then $q_{s}(s)$ can be found by performing the integral on the RHS of Eqn. 1. To achieve this at White Sands, we assumed flux only happens on the dunes and there is no flux into them from upwind. We identified dunes as non-flat regions in the transect for either DTM that changed elevation downwind of the roughness transition (Figs. S2 and S3).

\section{References}

Durán, O., Claudin, P., \& Andreotti, B. (2011). On aeolian transport: Grain-scale interactions, dynamical mechanisms and scaling laws. Aeolian Research, 3(3), 243270. 


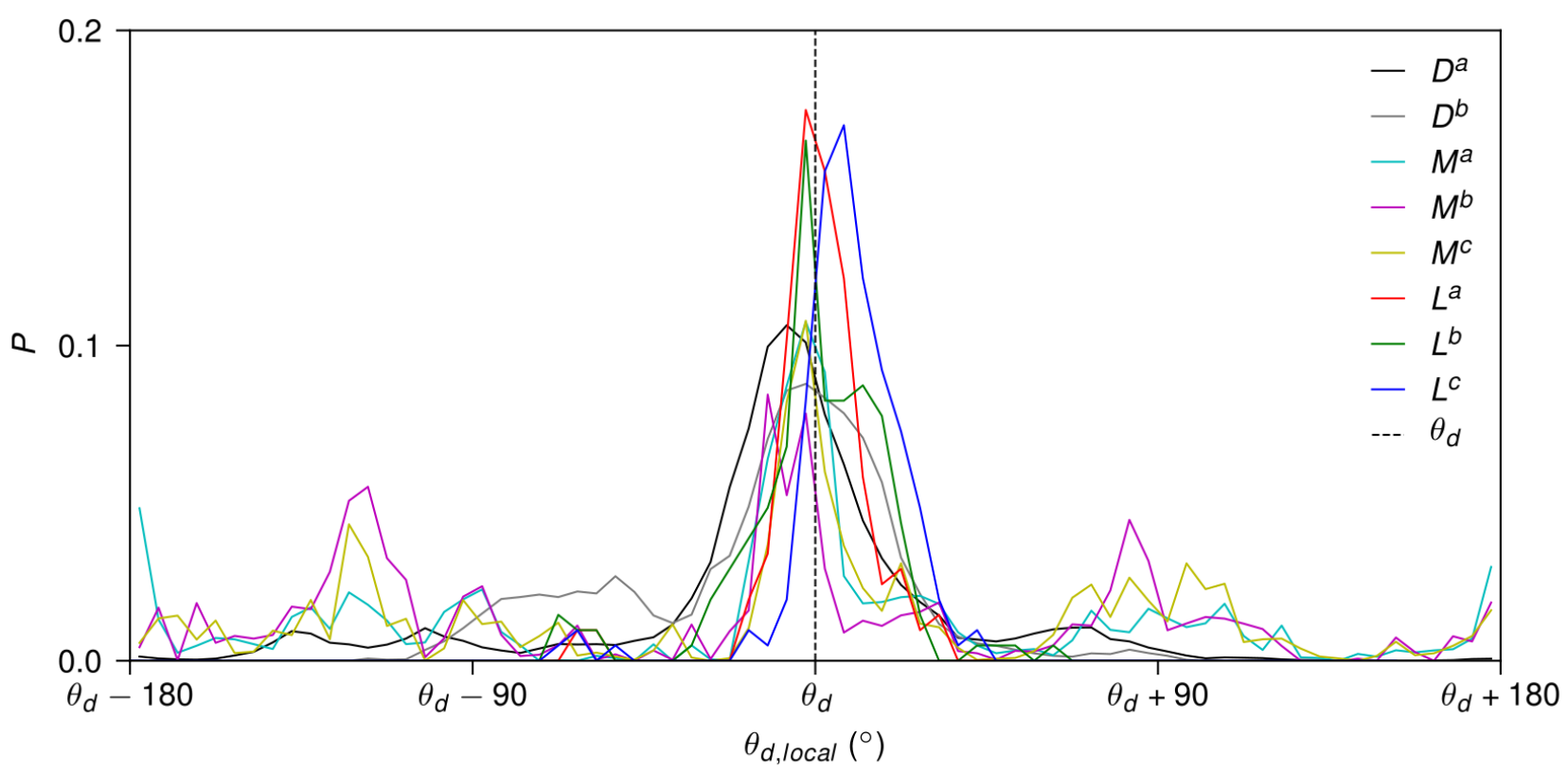

Figure S1. Probability distributions of dune direction relative to the determined dune direction $\theta_{d}$ calculated from 8 independent methods. Superscripts in legend refer to doppler wind lidar, $D$, meteorological tower $M$, and lidar DTM L. Subscripts are shown in Figure 1. Dune migration direction is best inferred by winds by first converting the wind vectors to sediment flux vectors. The wind measurements are all taken at $10 \mathrm{~m}$ elevation and are converted to flux using Law of the Wall with roughness $z_{0, \text { in }}$ and relationship to $u_{*}$ from Durán et al. (2011) (Eqn. 6). The dune direction distribution from the DTMs is found by local dune directions inferred from dune slip-face orientations (see Figure S2). This plot illustrates that $\theta_{d}=25^{\circ}$ (counterclockwise from East) is a reasonable global dune direction for White Sands. 

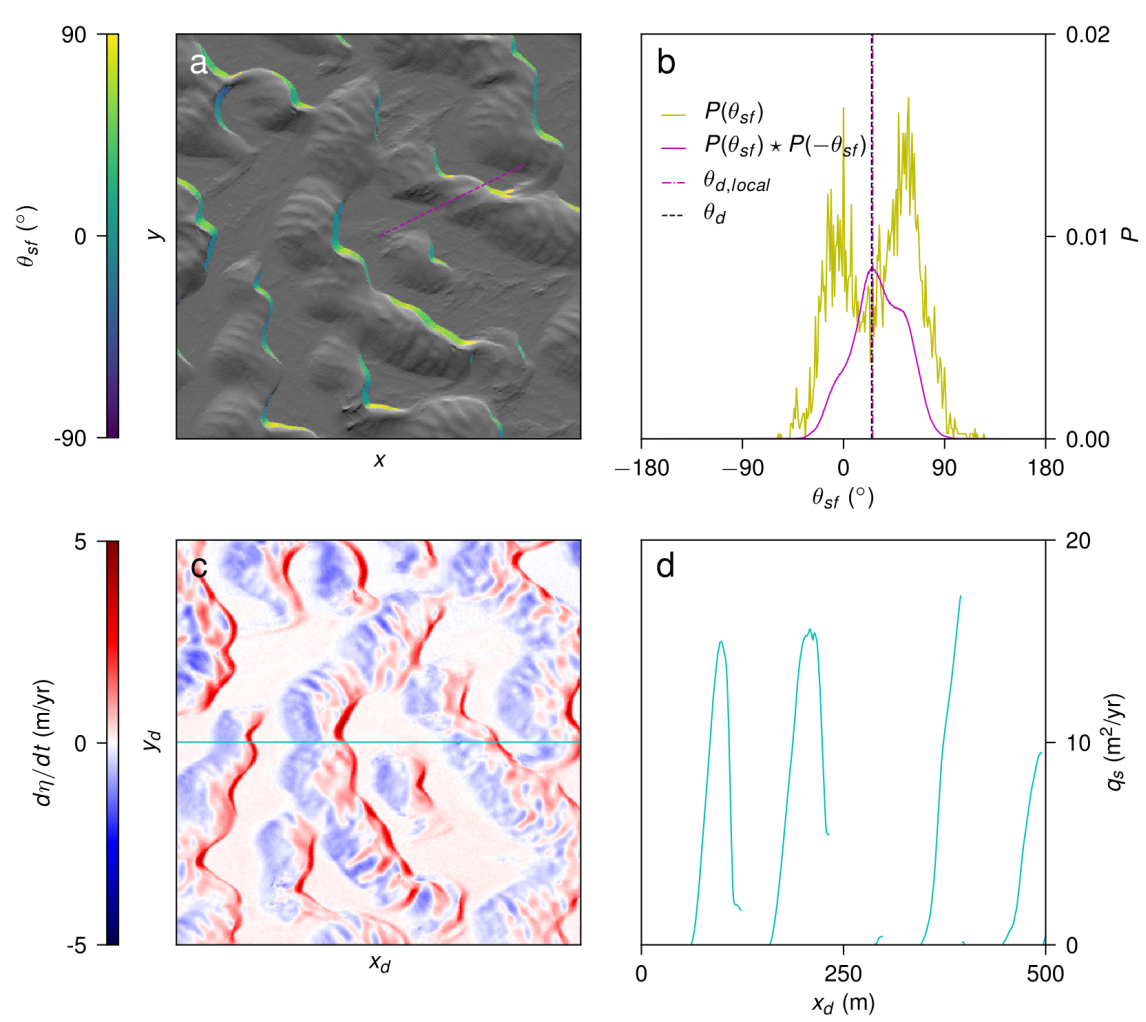

Figure S2. An illustration of the method used to find dune direction and sediment flux from the DTMs on a $500 \mathrm{~m}^{2}$ domain. (a) A 'hill-shade map' (where $x$ is West-East and $y$ is South-North) with the slip-faces highlighted and colored by their orientations, $\theta_{s f}$. Slip faces are found by being near the angle of repose. Overlaid is the inferred dune direction for this domain found in (b) extending from the domain center (magenta dashed line). (b) The probability distribution of slipface orientations highlighted in (a), $P\left(\theta_{s f}\right)$ (yellow), the cross-correlation of this distribution with itself flipped in the x-axis, $P\left(\theta_{s f}\right) \star P\left(-\theta_{s f}\right)$ (magenta), the angle of maximum cross-correlation, $\theta_{d, l o c a l}$ (magenta dash-dotted line), and the global dune direction, $\theta_{d}$ (black dashed line). This is defined as the local dune orientation. (c) A domain of the same size and center as (a) now rotated to the dune orientation axis $\left[x_{d}, y_{d}\right]$ and colored by the rate of elevation change between DTM pairs $d \eta / d t$, overlaid is the transect (cyan) analyzed in (d). (d) Sediment flux, $q_{s}$, along the transect of $d \eta / d t$ in (c) calculated using Eqn 5 . 


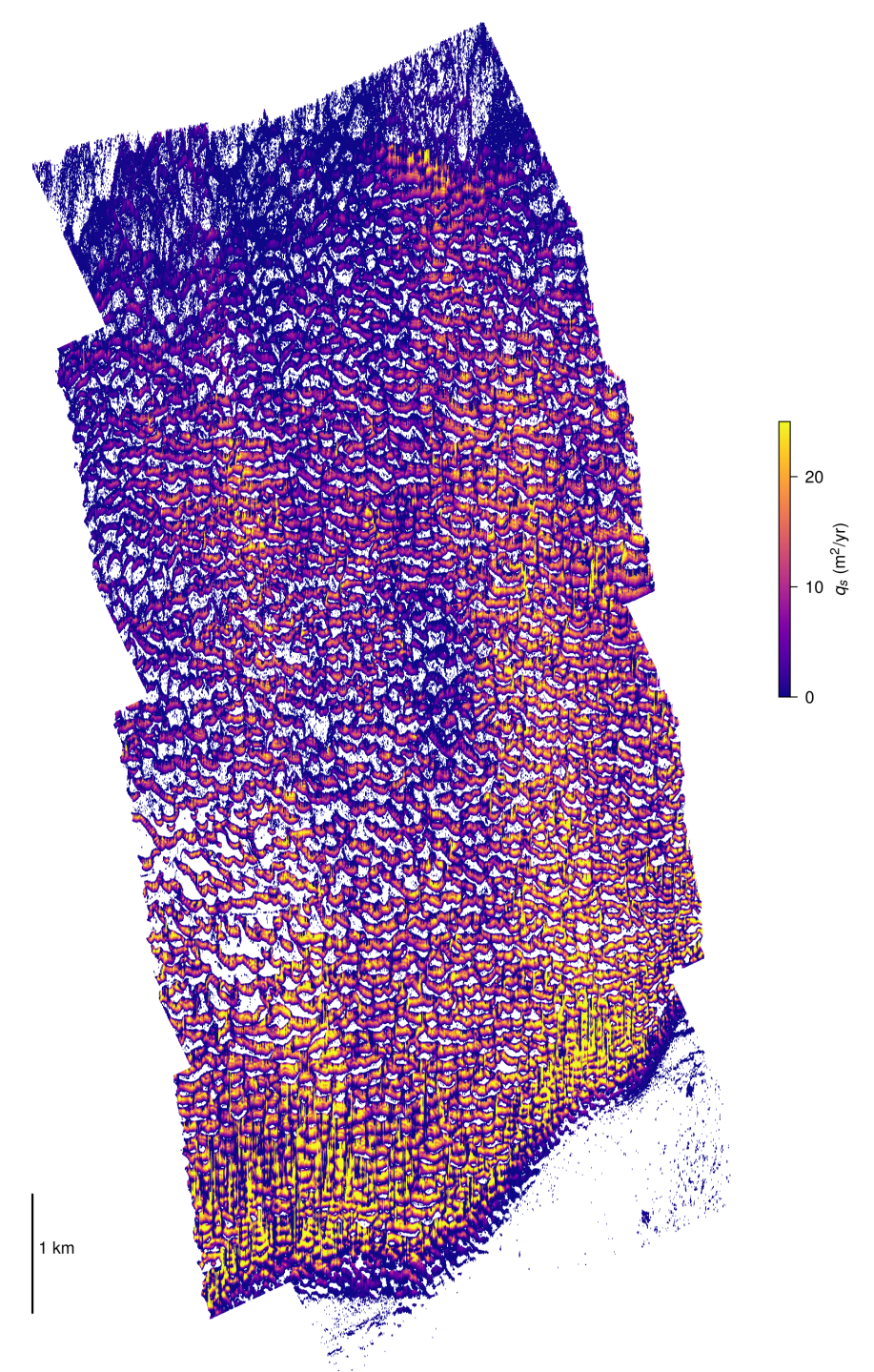

Figure S3. Sediment flux, $q_{s}$, map from the January and September 2009 DTMs. Across-page is $x_{d}$, Up-page is $y_{d}$, and a scale is given (black line). White regions are outside the domain, or regions not included in the flux calculations because they are (or are within $10 \mathrm{~m}$ of in $x_{d}$ ) nearly flat (slope $<0.01)$ in either DTM or they have spuriously large elevation changes $(>7 \mathrm{~m} / \mathrm{yr})$. We suspect the stream-wise bands of large sediment flux are an artifact of mismatched airplane flight paths. 

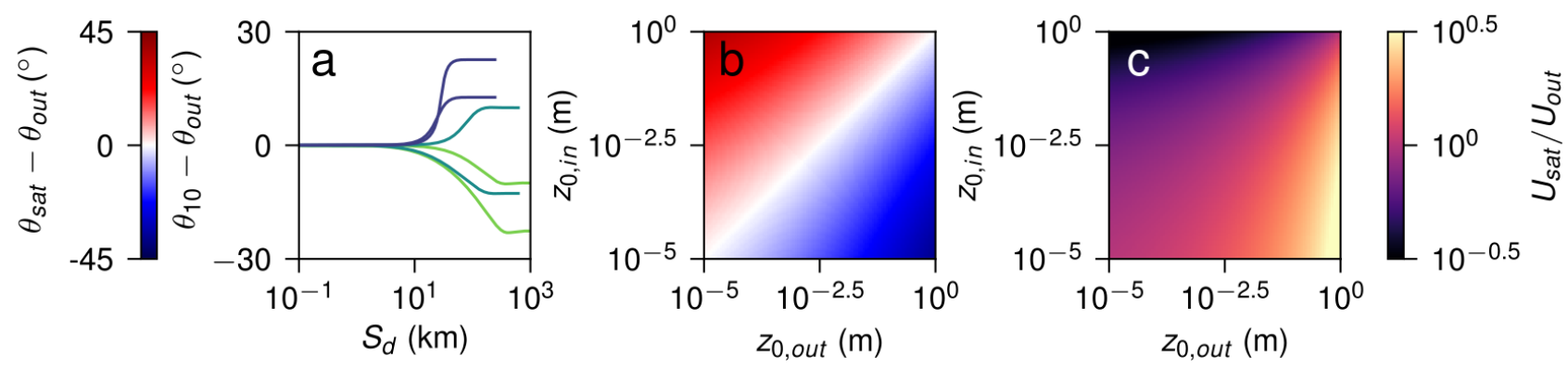

Figure S4. Extended theoretical results from Fig. 4. (a) Changes in the 10-m wind direction $\theta_{10}-\theta_{\text {out }}$ downwind of the roughness transition $S_{r}$ for different roughness pair scenarios (same as those in Figure 4a). (b) Veering angle between equilibrium winds, $\theta_{\text {sat }}-\theta_{\text {out }}$, colored on the left color-bar based on $z_{0, \text { in }}$ and $z_{0, \text { out }}$ pairs. Note that the absolute value of the veering angle also depends on latitude (here chosen as White Sands'), and the sign depends on the hemisphere (here chosen as north) where positive is 'bending to the left'. (c) Ratio of the equilibrium wind speeds, $U_{\text {sat }} / U_{\text {in }}$, colored in log-scale on the right color-bar. In the idealized equilibrium limit, flow crossing onto smoother terrain in the northern hemisphere will accelerate and bend to the right, for example. 